NBER WORKING PAPER SERIES

\title{
TOWARD AN EFFICIENCY RATIONALE FOR THE PUBLIC PROVISION OF PRIVATE GOODS
}

\author{
Hanming Fang \\ Peter Norman \\ Working Paper 13827 \\ http://www.nber.org/papers/w13827
NATIONAL BUREAU OF ECONOMIC RESEARCH
1050 Massachusetts Avenue
Cambridge, MA 02138
February 2008

We thank Bill Zame as well as many seminar and conference participants for helpful comments. All remaining errors are ours. The views expressed herein are those of the author(s) and do not necessarily reflect the views of the National Bureau of Economic Research.

NBER working papers are circulated for discussion and comment purposes. They have not been peerreviewed or been subject to the review by the NBER Board of Directors that accompanies official NBER publications.

(C) 2008 by Hanming Fang and Peter Norman. All rights reserved. Short sections of text, not to exceed two paragraphs, may be quoted without explicit permission provided that full credit, including @ is given to the source. 
Toward an Efficiency Rationale for the Public Provision of Private Goods

Hanming Fang and Peter Norman

NBER Working Paper No. 13827

February 2008

JEL No. D61,D82,H21,H42

\begin{abstract}
$\underline{\text { ABSTRACT }}$
This paper shows that public provision of private goods may be justified on pure efficiency grounds in an environment where individuals consume both public and private goods. The government's involvement in the provision of private goods provides it with information about individuals' private good purchases that facilitates more efficient revenue extraction for the provision of public goods. We show that public provision of the private good improves economic efficiency under a condition that is always fulfilled under stochastic independence and satisfied for an open set of joint distributions. Our model is an example where there is efficiency loss from separating revenue and expenditure problems in public finance, and is therefore of more general interest for the study of optimal taxation.
\end{abstract}

\author{
Hanming Fang \\ Department of Economics \\ Duke University \\ 213 Social Sciences Building \\ Box 90097 \\ Durham, NC 27708-0097 \\ and NBER \\ hanming.fang@duke.edu \\ Peter Norman \\ Department of Economics \\ University of North Carolina at Chapel Hill \\ 107 Gardner Hall \\ Chapel Hill, NC 27599-3305 \\ normanp@email.unc.edu
}




\section{Introduction}

All governments in developed countries not only provide public goods, but also devote considerable resources to the provision of private goods such as health care, housing, day care and education. This paper provides a novel efficiency rationale for such public provision of private goods, based on ideas from the literature on commodity bundling.

We model the government as a benevolent social planner who seeks to maximize the expected social surplus in an economy where citizens value a (binary) excludable public good, a (binary) private good, and where utility can be transferred using a perfectly divisible commodity called "money." The key friction is that citizens' valuations for the private and public goods are their private information. Accordingly, we require that the government restrict attention to provision mechanisms that are incentive compatible. In addition, we impose budget balance and individual rationality constraints.

We consider the optimal mechanism under two cases that differ in whether the government can keep track of individuals' purchases of the private good and use that information in constructing fees and access rules for the public good.

As a benchmark, we consider what we call the "separate provision" case, under which the planner designs provision mechanisms for the public and private goods separately. It is useful to imagine this as a setup where two government agencies are separately in charge of the public and private good provisions, even though they share a single budget constraint. The key restriction is that the two agencies are not allowed to share information about an individual's purchase in the other agency, otherwise any feasible provision mechanism can be used. In this case, the mechanism design problem simplifies to a problem where the planner sets an access fee for the public good, a provision probability of the public good, and a price for the private good. The solution to this problem has a flavor of Ramsey pricing, and the price of the private good is always set above its marginal cost of production. This is because the public good is generally under-provided due to the informational constraints, implying that a sufficiently small "tax" on the private good (which is the wedge between the the marginal cost for the private good and its price) leads to a second-order

efficiency loss in the private good market, but generates a first-order efficiency gain due to the increased probability of public good provision. A similar logic also delivers a less obvious result. There should always be a strictly positive access fee for the public good, thus a set of types with strictly positive measure will be excluded from usage. Moreover, we show that whenever the public good is desirable in a first-best world, it will be provided with positive probability in the optimal 
separate provision mechanism, with some of its cost covered by the "taxes" collected from the private goods.

The separate provision case is then contrasted with what we refer to as the "joint provision" case, where we allow the social planner to use all available information for decisions on both goods. The key difference from the separate provision case is that here a single government agency is in charge of the provision of both the public and private goods, and this agency can therefore condition the pricing and access rules for the public good on information revealed in the private good market. We are primarily interested in the qualitative question of whether joint provision mechanisms can improve expected social welfare over the optimal separate provision mechanisms.

The multidimensional nature of the joint provision mechanism design problem prevents us from characterizing the optimal joint provision mechanism. As a result, we follow McAfee et al. (1989) and consider "local deviations" from the optimal separate provision mechanism we earlier characterized. We show that, under some conditions, there always exists a joint provision mechanism that improves upon the optimal separate provision mechanism in a subspace of the feasible joint provision mechanisms. The particular subspace we consider consists of mechanisms fully characterized by three fixed prices, one for the private good, one for the public good, and one for the bundle consisting of both goods. Within this restricted set of feasible joint provision mechanisms, we provide a sufficient conditions for when there exist mechanisms in which the price of the private good an individual pays depends on whether she also consumes the public good that generate a higher social welfare than the optimal separate provision mechanism 11 Importantly, our sufficient condition is fulfilled under stochastic independence between the valuations of the two goods, and thus satisfied by an open set of joint distributions.

The above result can be interpreted as a new efficiency rationale for the public provision of private goods. The justification for such an interpretation is that in order to implement such a pricing policy, it is necessary that the planner keeps track of which individuals purchase the private good and somehow tax them on the basis of whether they also consume the public good. One practical way to achieve this is to allow all agents who opt into (by paying taxes) the "government bundle" access to both the private and the public good, while those who only want to consume the private good pay a separate and different price for the private good alone. This is consistent with

\footnotetext{
${ }^{1}$ It is well-known from Adams and Yellen (1976) and McAfee et al. (1989) that bundling can increase revenue for a multiproduct monopolist. Fang and Norman (2008) showed that it can also improve social welfare for the case of multiple public good provision. The current paper extends this intuition further to the case of bundling public and private goods.
} 
some important features of local public finance in the U.S.: first, residents who pay property taxes can enjoy the services, many of which has the nature of private goods (e.g., public schools), provided by the local government "for free"; second, most public universities charge different amounts of tuition for in-state or out-of-state residents 2

Our paper makes two major contributions to the existing literature on why it may be desirable to provide certain private goods publicly $3^{3}$ First, the role played by public provision of private goods in our paper differs substantially from explanations emphasized in the existing literature. In our paper, the government is involved in the provision of private goods because such involvement provides it with information of the private good demands of the individuals, which in turns allows the social planner to more efficiently extract revenues for the provision of public goods. This explanation has more in common with the literature on commodity bundling than the previous literature. In particular, we depart from all existing efficiency based explanations in that we do not rely on preferences for redistribution. This feature of our model is particularly desirable because several important publicly-provided private goods have neutral or regressive distributional effects.

Second, our paper imposes considerably less restrictions than the existing literature on the set of admissible policies in establishing our results. The benchmark outcome we use for comparison is the outcome that can be implemented under the optimal separate provision mechanism; this is in stark contrast to the existing literature where the benchmark outcome is often derived under various implicit restrictions on the market operations. The joint provision mechanism that improves upon the benchmark outcome in our model does require that consumers cannot engage in arbitrage. This restriction, however, seems reasonably descriptive of many publicly-provided private goods that we see in reality. For example, in-state college tuition, public health insurance, day care and public schools are all commodities that are difficult to resell, thus limiting arbitrage. This is important because our model thus also provides an explanation of why some, but not all, private goods are publicly provided.

Our analysis also illustrates a more general point, which is of relevance for the literature on optimal taxation and the traditional division of public economics into taxation and expenditure as separate subfields. Since Ramsey (1927), it has been standard in the optimal taxation literature to ask how to best raise a given target revenue without considering what the tax revenue is intended

\footnotetext{
${ }^{2}$ In our setup, the in-state tuition can be interpreted as the price of the private good (college education) for those who also purchase public good; and out-of-state tuition is the price for those who purchase the private good alone.

${ }^{3}$ We review the existing literature on the public provision of private goods in Section 2
} 
for. Our model is a simple yet plausible example where this standard approach is no longer valid, since the most efficient way to distribute and finance the public good in our model must be jointly determined $4^{4}$ In other words, the optimal commodity tax to finance the (excludable) public good depends on whether the consumer gets access to the public good. Hence, the analytically convenient dichotomy between government expenditures and revenue comes with an efficiency loss.

The remainder of the paper is structured as follows. Section 2 reviews the related literature on the public provision of private goods; Section 3 presents the model; Section 4 characterizes the optimal separate provision mechanism; Section 5 presents our main results regarding the optimality of public provision of private goods; and finally Section 6 concludes.

\section{Related Literature}

It is useful to divide the existing literature on public provision of private goods into three strands. The first strand, as represented by Fernandez and Rogerson (1995) and Epple and Romano (1996), is a positive theory of the public provision of private goods driven by political economy considerations $5^{5}$ Epple and Romano (1996) consider a political economy model where the level of a private good (say, "health care") and whether private purchases are permitted to supplement the public provision are both determined by majority voting. They show that, under standard assumptions on voter preferences, a regime with positive government provision and no restriction on private supplement is majority preferred to a regime of either only market provision or only government provision. The key intuition is that the median voter, which typically has income below the mean in the population, will receive positive transfers in equilibrium from richer citizens if private goods are publicly provided. Fernandez and Rogerson (1995) use a similar model, with the crucial difference that the private good is only partially subsidized, to show that it is also possible to explain public provision of private goods with regressive distributional consequences. While the political economy considerations in these models are plausible and possibly important for a positive understanding of why government provides private goods, their results do hinge crucially on the restrictions imposed on the policy instruments; that is, what is not explained is why transfers must be in-kind (in the form of publicly provided private goods) instead of pure cash transfers ${ }^{6}$

\footnotetext{
${ }^{4}$ Related points are made in Boadway et al. (1998) and Blomquist and Christiansen (2007).

${ }^{5}$ See also Bergstrom and Blomquist (1996) and Gouveia (1997).

${ }^{6}$ However, see Blomquist and Christiansen (1999) for a model where efficiency gains of in-kind transfers are combined with a political economy setup.
} 
The second strand assumes that the government has a preference for redistribution and shows that public provision of private goods may serve as a tool for redistribution from the rich to the poor. As in the first strand of the literature, it also assumes, instead of explains, why in-kind transfers are preferred to cash transfers. For example, Besley and Coate (1991) considered the case where the publicly provided private goods are discrete. In their model, if a household is dissatisfied with the (uniform) quality of publicly provided private goods, it opts out and purchases a higher quality version from the market. If the quality demand increases with income, mainly rich households opt out from the publicly provided private goods, implying that the system of public provision of private goods can serve as a transfer towards less wealthy individuals..$^{7}$ As Besley and Coate (1991) acknowledged in their concluding remarks, there may exist other feasible policies that can achieve the same distributional goals more efficiently if one does not restrict attention to in-kind transfers 8

The third strand, to which our paper is most closely related, takes a normative perspective and identifies circumstances under which providing a private good in-kind is a more efficient transfer instrument than cash transfers. Blackorby and Donaldson (1988) considered an environment where consumers have private information about their preferences and showed that in-kind transfers can be desirable due to its screening role, which allows better targeted transfers.$^{9}$ Coate (1995) considered an environment where the rich has altruistic preferences towards the poor and would like to insure the poor's income risks. If cash assistance is given to the poor, the poor may have incentives not to purchase insurance and exploit the well-know Samaritan's Dilemma. As a result, the rich may instead prefer to directly provide insurance to the poor. His explanation crucially relies on the rich's lack of commitment not to help the poor if the poor does not purchase insurance with the cash assistance ${ }^{10}$ Garratt and Marshall (1994) considers the case of the public financing of college education. They argue that public financing of college education (which is a private good) provides gambles that families desire. Their explanation implicitly relies on the unmodeled notion that private market can not provide such lotteries demanded by the families.

Our paper complements the above literature by analyzing the full mechanism design problem faced by a social planner when individuals have private information about their preferences. Notably, we do not impose any exogenous restrictions on the set of feasible policy instruments other

\footnotetext{
${ }^{7}$ Other arguments based on a desire to redistribute income can be found in Blomquist and Christiansen (1995) and Cremer and Gahvari (1997).

${ }^{8}$ See Gahvari and Mattos (2007) for an interesting extension of Besley and Coate (1991).

${ }^{9}$ See also Nichols and Zeckhauser (1982) for similar arguments.

${ }^{10} \mathrm{~A}$ similar model is considered by Bruce and Waldman (1991).
} 
than the natural participation and incentive compatibility constraints in establishing our comparison benchmark of what can be achieve by the market in the absence of public provision of private goods. The key role played by the public provision of private goods in our paper also substantially differs from targeted transfer (Blackorby and Donaldson 1988), commitment (Coate 1988) and demand for gambles (Garratt and Marshall 1994). In our paper, the government's involvement in the provision of private goods provides it with information about individuals' private good purchases that facilitates more efficient revenue extraction for the provision of public goods. The feature of our model that the efficiency rationale for the public provision of private goods does not rely on redistributive preferences is particularly attractive because several important publicly-provided private goods have neutral or regressive distributional effects.

\section{The Environment}

Consider an economy populated by a continuum of ex ante identical consumers. Consumers have preferences over a binary and excludable public good, a binary private good and a perfectly divisible numeraire good that we will refer to as "money." The public good can be produced at a cost $K>0$; and the binary private good can be produced at unit cost $c>0$.

A consumer is characterized by $\left(\theta_{G}, \theta_{P}\right) \in \Theta_{G} \times \Theta_{P} \subseteq R^{2}$ where $\theta_{G}$ is her valuation for the public good and $\theta_{P}$ is her valuation for the private good. A consumer's valuations $\left(\theta_{G}, \theta_{P}\right)$ is her private information. We denote $F: \Theta_{G} \times \Theta_{P} \rightarrow[0,1]$ as the cumulative distribution over consumer types $\left(\theta_{G}, \theta_{P}\right)$, and write $F_{G}: \Theta_{G} \rightarrow[0,1]$ and $F_{P}: \Theta_{P} \rightarrow[0,1]$, respectively, as the marginal cumulative distribution of $\theta_{G}$ and $\theta_{P}$. Consumers are assumed to be risk neutral. The expected payoff of a consumer with type $\left(\theta_{G}, \theta_{P}\right)$ who consumes the public good with probability $\phi_{G}$ and the private good with probability $\phi_{P}$, and gives up (in expectation) $m$ units of money is given by

$$
u\left(\phi_{G}, \phi_{P}, m ; \theta_{G}, \theta_{P}\right)=\phi_{G} \theta_{G}+\phi_{P} \theta_{P}-m
$$

The reader may observe that we have built in the assumption that wealth constraints are always non-binding in (1). If $\overline{\theta_{G}}$ and $\overline{\theta_{P}}$ are upper bounds for $\theta_{G}$ and $\theta_{P}$ respectively, this assumption can be justified if every consumer has an endowment of money in excess of $\overline{\theta_{G}}+\overline{\theta_{P}}$. We assume that $c<\overline{\theta_{P}}$.

Remark 1. We have chosen to model the agents in the economy as a continuum in order to obtain a clean characterization of the optimal mechanism. The characterization of the constrained efficient 
mechanism is much more complicated in a finite population economy with private information. In particular, the optimal provision rule depends in a non-trivial way on the realized types. However, in a finite economy with sufficiently many agents, the effects of making the provision rule depend on announcements become negligible due to intuition similar to that of the "Paradox of Voting." As a result, the optimal mechanism can be approximated by a mechanism where no agent is pivotal to the decision 11 Hence, our convenient continuum assumption can be viewed as an approximation of a large finite economy ${ }^{12}$

Remark 2. However, the continuum specification has a few awkward technical aspects. In particular, we will treat the distribution $F$ as a cross-section of agents, which is necessary to interpret the right hand side of the balanced budget constraint $(\mathrm{BB})$ in Subsection 4.1 as the total revenue from the mechanism; at the same time, the distribution $F$ is also interpreted as the probability distribution over types for any individual agents. As is well-known, this is inconsistent with stochastic independence across agents in standard probability measures (see, however, Al-Najjar 2004 for a solution to the problem).

\section{Optimal Separate Provision Mechanisms}

In this section we analyze the benchmark case where the mechanism designer cannot condition either the price or the provision probability of one good on a consumer's reported valuation for the other good. The most obvious way to interpret this setup is that the "markets" for the private and public goods are physically separated in space and that the designer lacks the technology to track behavior of individual agents across markets. Formally:

Definition 1 A separate provision mechanism is a quadruple $\left(\phi_{G}, t, \phi_{P}, p\right)$, where:

- $\phi_{G}: \Theta_{G} \rightarrow[0,1]$ is the probability of consuming the public good;

- $t: \Theta_{G} \rightarrow \mathrm{R}$ is the fee for consuming the public good;

\footnotetext{
${ }^{11}$ See Norman (2004). Schmitz (1997) also makes essentially the same point in the context of monopolistic provision of an excludable public good.

${ }^{12}$ Note, however, that to get a limit characterization corresponding to the one in this paper, it is necessary to assume that per capita provision costs stay bounded away from zero as the number of participants goes to infinity. Otherwise, a pivot mechanism will work also with a large finite set of agents (see Hellwig 2003).
} 
- $\phi_{P}: \Theta_{P} \rightarrow[0,1]$ is the probability of consuming the private good;

- $p: \Theta_{P} \rightarrow \mathrm{R}$ is the fee for consuming the private good.

Note that both $\phi_{G}$ and $t$ are functions of the reported valuation for the public good only; and $\phi_{P}$ and $p$ are functions of the reported valuation of the private good only. As will be made clear below, our notion of separate provision mechanism still leaves room for cross-subsidization between the private and public goods, which is the only link allowed between the two design problems.

\subsection{The Planning Problem}

Given a separate provision mechanism, the expected utility for an agent of type $\left(\theta_{G}, \theta_{P}\right)$ is given by $\phi_{G}\left(\theta_{G}\right) \theta_{G}-t\left(\theta_{G}\right)+\phi_{P}\left(\theta_{P}\right) \theta_{P}-p\left(\theta_{P}\right)$. We assume that the planner seeks to maximize the ex ante expected utility of the representative consumer.

The objective function of the planner can be written as

$$
\begin{aligned}
& \int_{\Theta_{G}} \int_{\Theta_{P}}\left[\phi_{G}\left(\theta_{G}\right) \theta_{G}-t\left(\theta_{G}\right)+\phi_{P}\left(\theta_{P}\right) \theta_{P}-p\left(\theta_{P}\right)\right] d F\left(\theta_{G}, \theta_{P}\right) \\
= & \int_{\Theta_{G}}\left[\phi_{G}\left(\theta_{G}\right) \theta_{G}-t\left(\theta_{G}\right)\right] d F_{G}\left(\theta_{G}\right)+\int_{\Theta_{P}}\left[\phi_{P}\left(\theta_{P}\right) \theta_{P}-p\left(\theta_{P}\right)\right] d F_{P}\left(\theta_{P}\right) .
\end{aligned}
$$

Since types are assumed to be private information, agents must be willing to disclose their preferences to the planner. That is, it must be incentive compatible to report truth-fully,

$$
\begin{aligned}
& \phi_{G}\left(\theta_{G}\right) \theta_{G}-t\left(\theta_{G}\right)+ \phi_{P}\left(\theta_{P}\right) \theta_{P}-p\left(\theta_{P}\right) \geq \phi_{G}\left(\widehat{\theta_{G}}\right) \theta_{G}-t\left(\widehat{\theta_{G}}\right)+\phi_{P}\left(\widehat{\theta_{P}}\right) \theta_{P}-p\left(\widehat{\theta_{P}}\right), \\
& \forall\left(\theta_{G}, \theta_{P}\right),\left(\widehat{\theta_{G}}, \widehat{\theta_{P}}\right) \in \Theta_{G} \times \Theta_{P} .
\end{aligned}
$$

We also assume that agents must be willing to participate. Given the continuum-agent formulation, there is no distinction between interim and ex post participation constraints, and assuming that the non-participation utility is constant (and normalized to zero), we may write these constraints as

$$
\phi_{G}\left(\theta_{G}\right) \theta_{G}-t\left(\theta_{G}\right)+\phi_{P}\left(\theta_{P}\right) \theta_{P}-p\left(\theta_{P}\right) \geq 0, \quad \forall\left(\theta_{G}, \theta_{P}\right) \in \Theta_{G} \times \Theta_{P} .
$$

Finally, we assume that the planner must satisfy the natural feasibility constraint, namely that the total costs for the production of the public and the private goods should not exceed the total revenue collected from the agents. We may write this constraint as

$$
K\left[\sup _{\theta_{G} \in \Theta_{G}} \phi_{G}\left(\theta_{G}\right)\right]+\int_{\Theta_{P}} c \phi_{P}\left(\theta_{P}\right) d F_{P}\left(\theta_{P}\right) \leq \int_{\Theta_{G}} t\left(\theta_{G}\right) d F_{G}\left(\theta_{G}\right)+\int_{\Theta_{P}} p\left(\theta_{P}\right) d F_{P}\left(\theta_{P}\right) .
$$


To understand $(\mathrm{BB})$, note that, since the public good is non-rival, the cost is independent of the number of agents actually consuming the good. That is, it costs $K$ if the good is provided and 0 otherwise. Our formulation allows the mechanism designer to randomize between provision and non-provision. While $(\mathrm{BB})$ says that budget balances in expectation, it is easy to adjust transfers without changing the interim expected payoffs in such a way as to balance the budget for sure (see, e.g., Borgers and Norman 2008).

\subsection{Solving the Planning Problem}

First, note that an immediate implication of separate provision mechanism assumption is that a mechanism satisfies incentive compatibility constraints $(\mathrm{IC})$ if and only if

$$
\begin{aligned}
& \phi_{G}\left(\theta_{G}\right) \theta_{G}-t\left(\theta_{G}\right) \geq \phi_{G}\left(\widehat{\theta_{G}}\right) \theta_{G}-t\left(\widehat{\theta_{G}}\right) \quad \forall \theta_{G}, \widehat{\theta_{G}} \in \Theta_{G}, \\
& \phi_{P}\left(\theta_{P}\right) \theta_{P}-p\left(\theta_{P}\right) \geq \phi_{P}\left(\widehat{\theta_{P}}\right) \theta_{P}-p\left(\widehat{\theta_{P}}\right) \quad \forall \theta_{P}, \widehat{\theta_{P}} \in \Theta_{P},
\end{aligned}
$$

where (3) can be viewed as the incentive compatibility constraint for the public good provision and (4) is the incentive compatibility constraint for the private good provision. Hence, the separability restrictions make the model completely single-dimensional, and equivalent to a model where one set of agents care only for the public good and another set of agents care only about the private good.

Further note that, if $\left(\phi_{G}^{*}, t^{*}, \phi_{P}^{*}, p^{*}\right)$ is an optimal separate provision mechanism, then $\left(\phi_{G}^{*}, t^{*}\right)$ solves the following problem given $\left(\phi_{P}^{*}, p^{*}\right)$ :

$$
\begin{array}{ll} 
& \max _{\left(\phi_{G}, t\right): \Theta_{G} \rightarrow[0,1] \times \mathrm{R}}\left\{\int_{\Theta}\left[\phi_{G}\left(\theta_{G}\right) \theta_{G}-t\left(\theta_{G}\right)\right] d F_{G}\left(\theta_{G}\right)+\int_{\theta_{P}}\left[\phi_{P}^{*}\left(\theta_{P}\right) \theta_{P}-p^{*}\left(\theta_{P}\right)\right] d F_{P}\left(\theta_{P}\right)\right\} \\
\text { s.t. } & 0 \leq \phi_{G}\left(\theta_{G}\right) \theta_{G}-t\left(\theta_{G}\right)-\phi_{G}\left(\widehat{\theta_{G}}\right) \theta_{G}+t\left(\widehat{\theta_{G}}\right), \quad \forall \theta_{G}, \widehat{\theta_{G}} \in \Theta_{G}, \\
& 0 \leq \phi_{G}\left(\theta_{G}\right) \theta_{G}-t\left(\theta_{G}\right)+\phi_{P}^{*}\left(\theta_{P}\right) \theta_{P}-p^{*}\left(\theta_{P}\right), \quad \forall\left(\theta_{G}, \theta_{P}\right) \in \Theta_{G} \times \Theta_{P}, \\
& 0 \leq \int_{\Theta_{G}} t\left(\theta_{G}\right) d F_{G}\left(\theta_{G}\right)+\int_{\Theta_{P}} p^{*}\left(\theta_{P}\right) d F_{P}\left(\theta_{P}\right)-K\left[\sup _{\theta_{G} \in \Theta_{G}} \phi_{G}\left(\theta_{G}\right)\right]-\int_{\Theta_{P}} c \phi_{P}^{*}\left(\theta_{P}\right) d F_{P}\left(\theta_{P}\right) ;
\end{array}
$$

and $\left(\phi_{P}^{*}, p^{*}\right)$ solves the following problem given $\left(\phi_{G}^{*}, t^{*}\right)$ :

$$
\begin{array}{ll} 
& \max _{\left(\phi_{P}, p\right): \Theta_{P} \rightarrow[0,1] \times \mathrm{R}}\left\{\int_{\Theta_{G}}\left[\phi_{G}^{*}\left(\theta_{G}\right) \theta_{G}-t^{*}\left(\theta_{G}\right)\right] d F_{G}\left(\theta_{G}\right)+\int_{\Theta_{P}}\left[\phi_{P}\left(\theta_{P}\right) \theta_{P}-p\left(\theta_{P}\right)\right] d F_{P}\left(\theta_{P}\right)\right\} \\
\text { s.t. } & 0 \leq \phi_{P}\left(\theta_{P}\right) \theta_{P}-p\left(\theta_{P}\right)-\phi_{P}\left(\widehat{\theta_{P}}\right) \theta_{P}+p\left(\widehat{\theta_{P}}\right) \quad \forall \theta_{P}, \widehat{\theta_{P}} \in \Theta_{P} \\
& 0 \leq \phi_{G}^{*}\left(\theta_{G}\right) \theta_{G}-t^{*}\left(\theta_{G}\right)+\phi_{P}\left(\theta_{P}\right) \theta_{P}-p\left(\theta_{P}\right) \quad \forall\left(\theta_{G}, \theta_{P}\right) \in \Theta_{G} \times \Theta_{P} \\
& 0 \leq \int_{\Theta_{G}} t^{*}\left(\theta_{G}\right) d F_{G}\left(\theta_{G}\right)+\int_{\Theta_{P}} p\left(\theta_{P}\right) d F_{P}\left(\theta_{P}\right)-K\left[\sup _{\theta_{G} \in \Theta_{G}} \phi_{G}^{*}\left(\theta_{G}\right)\right]-\int_{\Theta_{P}} c \phi_{P}\left(\theta_{P}\right) d F_{P}\left(\theta_{P}\right) .
\end{array}
$$

Both (5) and (8) are problems that can be solved using standard techniques going back to Myerson's 
(1981) analysis of optimal auction design. Define the "indirect utility functions"

$$
\begin{aligned}
& U_{G}\left(\theta_{G}\right) \equiv \theta_{G} \phi_{G}\left(\theta_{G}\right)-t\left(\theta_{G}\right), \\
& U_{P}\left(\theta_{P}\right) \equiv \theta_{P} \phi_{P}\left(\theta_{P}\right)-p\left(\theta_{P}\right) .
\end{aligned}
$$

A routine argument (see, e.g. Mas-Colell et al. 1995, page 888) can be used to establish the following lemmas:

Lemma 1 Suppose that $\Theta_{G}=\left[\underline{\theta_{G}}, \overline{\theta_{G}}\right]$ and that the marginal density $f_{G}\left(\theta_{G}\right)$ is strictly positive on its support. Then, $\left(\phi_{G}, t\right)$ satisfies the incentive compatibility constraints (6) if and only if $\phi_{G}$ is weakly increasing in $\theta_{G}$ and

$$
U_{G}\left(\theta_{G}\right)=U_{G}\left(\widehat{\theta_{G}}\right)+\int_{\widehat{\theta_{G}}}^{\theta_{G}} \phi_{G}(x) d x \quad \forall \theta_{G}, \widehat{\theta_{G}} \in \Theta_{G} .
$$

Lemma 2 Suppose that $\Theta_{P}=\left[\underline{\theta_{P}}, \overline{\theta_{P}}\right]$ and that the marginal density $f_{P}\left(\theta_{P}\right)$ is strictly positive on its support. Then, $\left(\phi_{P}, p\right)$ satisfies the incentive compatibility constraints (9) if and only if $\phi_{P}$ is weakly increasing in $\theta_{P}$ and

$$
U_{P}\left(\theta_{P}\right)=U\left(\widehat{\theta_{P}}\right)+\int_{\widehat{\theta_{P}}}^{\theta_{P}} \phi_{P}(x) d x \quad \forall \theta_{P}, \widehat{\theta_{P}} \in \Theta_{P}
$$

Equally routine procedures (see, e.g. Mas-Colell et al. 1995, page 890), using Lemmas 1 and 2. show that the aggregate transfer revenues from the public goods fees and the private goods fees respectively can be determined purely in terms of the utility of the lowest type and the provision rules as

$$
\begin{aligned}
\int_{\Theta_{G}} t\left(\theta_{G}\right) d F_{G}\left(\theta_{G}\right) & =\int_{\Theta_{G}} \phi_{G}\left(\theta_{G}\right)\left(\theta_{G}-\frac{1-F_{G}\left(\theta_{G}\right)}{f_{G}\left(\theta_{G}\right)}\right) d F_{G}\left(\theta_{G}\right)-U_{G}\left(\underline{\theta_{G}}\right) \\
\int_{\Theta_{P}} p\left(\theta_{P}\right) d F_{P}\left(\theta_{P}\right) & =\int_{\Theta_{P}} \phi_{P}\left(\theta_{P}\right)\left(\theta_{P}-\frac{1-F_{P}\left(\theta_{P}\right)}{f_{P}\left(\theta_{P}\right)}\right) d F_{P}\left(\theta_{P}\right)-U_{P}\left(\underline{\theta_{P}}\right) .
\end{aligned}
$$

We also note that, due to the existence of the numeraire good "money," we can without loss of generality assume that the participation constraint of type $\left(\underline{\theta_{G}}, \underline{\theta_{P}}\right)$ binds:

Lemma 3 Suppose that $\left(\phi_{G}^{*}, t^{*}, \phi_{P}^{*}, p^{*}\right)$ is an optimal separate provision mechanism. Then there exists $(\hat{t}, \hat{p})$ such that $\left(\phi_{G}^{*}, \hat{t}, \phi_{P}^{*}, \hat{p}\right)$ is an optimal separate provision mechanism and

$$
\phi_{G}^{*}\left(\underline{\theta_{G}}\right) \underline{\theta_{G}}-\hat{t}\left(\underline{\theta_{G}}\right)+\phi_{P}^{*}\left(\underline{\theta_{P}}\right) \underline{\theta_{P}}-\hat{p}\left(\underline{\theta_{P}}\right)=0
$$


Since all higher types can mimic $\left(\underline{\theta_{G}}, \underline{\theta_{P}}\right)$, incentive compatibility automatically implies that the participation constraints hold for all higher types, provided that it is satisfied for type $\left(\underline{\theta_{G}}, \underline{\theta_{P}}\right)$. Using (11) and Lemma 3, we can therefore reformulate (5) as

$$
\begin{aligned}
& \max _{\phi_{G}: \Theta_{G} \rightarrow[0,1]} \int_{\Theta_{G}}\left[\phi_{G}\left(\theta_{G}\right) \theta_{G}\right] d F_{G}\left(\theta_{P}\right)-K \phi_{G}\left(\overline{\theta_{G}}\right) \\
\text { s.t. } 0 \leq & \int_{\Theta_{G}} \phi_{G}\left(\theta_{G}\right)\left(\theta_{G}-\frac{1-F_{G}\left(\theta_{G}\right)}{f_{G}\left(\theta_{G}\right)}\right) d F_{G}\left(\theta_{G}\right)-K \phi_{G}\left(\overline{\theta_{G}}\right) \\
& +\int_{\Theta_{P}}\left[p^{*}\left(\theta_{P}\right)-c \phi_{P}^{*}\left(\theta_{P}\right)\right] d F_{P}\left(\theta_{P}\right) \\
0 \leq & \phi_{G}\left(\theta_{G}\right) \leq 1 \text { for all } \theta_{G} \\
& \phi_{G}(\cdot) \text { is weakly increasing. }
\end{aligned}
$$

To understand the objective function, observe that the social feasibility constraint (7) must bind. The objective function (13) is thus simply obtained by substitution of (7) into the objective function of the problem, eliminating the constants, and noting, by the fact that $\phi_{G}$ is monotonic, that $\sup _{\theta_{G} \in \Theta_{G}} \phi_{G}\left(\theta_{G}\right)=\phi_{G}\left(\overline{\theta_{G}}\right)$. The integral constraint $(14)$, together with the monotonicity requirement (16), combines all incentive and participation constraints; and the constraints in (15) are the boundary constraints for the provision probabilities.

To facilitate further interpretations, it may be useful to observe that the problem for a profit maximizing monopolist for the public good would be to maximize

$$
\int_{\Theta_{G}} \phi_{G}\left(\theta_{G}\right)\left(\theta_{G}-\frac{1-F_{G}\left(\theta_{G}\right)}{f_{G}\left(\theta_{G}\right)}\right) d F_{G}\left(\theta_{G}\right)-K \phi_{G}\left(\overline{\theta_{G}}\right)
$$

subject only to the constraints 15 and (16). For this problem, the "no-haggling" logic of Stokey (1979), Myerson (1981) and Riley and Zeckhauser (1983) immediately implies that the profitmaximizing mechanism is, without loss of generality, one where the monopolist charges a single price ${ }^{13}$ However, this result does not extend to our problem where profits appear as a constraint in (14). In general, the solution to the problem 13 may very well be a randomized mechanism. ${ }^{14}$ However, randomizations can be ruled out by making an additional restriction on the distribution of types. Define

$$
x_{G}\left(\theta_{G}\right) \equiv \theta_{G}-\frac{1-F_{G}\left(\theta_{G}\right)}{f_{G}\left(\theta_{G}\right)},
$$

\footnotetext{
${ }^{13}$ If there is a profit-maximizing random mechanism, then a single price mechanism that charges any price in the support of the randomized mechanism is also optimal.

${ }^{14}$ This is easy to realize by considering the case with two types, $\theta_{G}^{l}$ and $\theta_{G}^{h}$. Assuming that charging a flat fee equal to $\theta_{G}^{l}$ would violate the budget constraint, whereas charging $\theta_{G}^{h}$ would give a strict surplus, it is obvious that the surplus can be made higher by letting the low type agents consume with some probability. The example can easily be extended to continuous densities.
} 
which is often referred to as the "virtual surplus." We can then show the following result:

Lemma 4 Suppose that $x_{G}\left(\theta_{G}\right)$ as defined in (18) is weakly increasing in $\theta_{G}$ and that $\phi_{G}^{*}$ is a solution to 1 (13). Then there exists some $t^{*}$ such that

$$
\phi_{G}^{*}\left(\theta_{G}\right)=\left\{\begin{array}{cl}
\phi_{G}\left(\overline{\theta_{G}}\right) & \text { for } \theta_{G} \geq \frac{t^{*}}{\phi_{G}\left(\overline{\theta_{G}}\right)} \\
0 & \text { for } \theta_{G}<\frac{t^{*}}{\phi_{G}\left(\overline{\theta_{G}}\right)}
\end{array}\right.
$$

Lemma 4 follows almost immediately from the fact that $\phi_{G}\left(\theta_{G}\right)$ appears linearly in both the objective function and the constraint. Thus, characterizing the solution to (13) is reduced to determining two variables: (1) the probability of public good provision $\phi_{G}\left(\overline{\theta_{G}}\right)$; and (2) a user fee (or equivalently, a threshold valuation for being allowed to consume the good when it is produced) ${ }^{15}$

In the same spirit, the private goods problem (8) may be reformulated as

$$
\begin{aligned}
& \max _{\left(\phi_{P}\right): \Theta_{P} \rightarrow[0,1]} \int_{\Theta_{P}}\left[\phi_{P}\left(\theta_{P}\right)\left(\theta_{P}-c\right)\right] d F_{P}\left(\theta_{P}\right) \\
\text { s.t. } 0 \leq & \int_{\Theta_{P}} \phi_{P}\left(\theta_{P}\right)\left[\theta_{P}-\frac{1-F_{P}\left(\theta_{P}\right)}{f_{P}\left(\theta_{P}\right)}-c\right] d F_{P}\left(\theta_{G}\right) \\
& +\int_{\Theta_{G}} t^{*}\left(\theta_{G}\right) d F_{G}\left(\theta_{G}\right)-K \phi_{G}^{*}\left(\overline{\theta_{G}}\right) \\
0 \leq & \phi_{P}\left(\theta_{P}\right) \leq 1 \text { for all } \theta_{P} \\
& \theta_{P}(\cdot) \text { is weakly increasing. }
\end{aligned}
$$

Again the objective function is derived by substituting the (binding) budget constraint into the objective function of Problem (8) and eliminating constants. A similar argument allows us to conclude that all we need to do is to find a price to charge for the private good. Again, the fact that 20 is not a profit-maximization problem makes it necessary to make regularity assumptions on the virtual surplus in order to rule out a randomized optimal mechanism. Define

$$
x_{P}\left(\theta_{P}\right)=\theta_{P}-\frac{1-F_{P}\left(\theta_{P}\right)}{f_{P}\left(\theta_{P}\right)} .
$$

We have the following result:

\footnotetext{
${ }^{15}$ The continuum-consumer assumption in itself trivializes the provision decision in the sense that this can no longer be made contingent on the realized distribution of types. However, we still need to make a (standard) regularity assumption in order to obtain the fixed price characterization. See Norman (2004) for an assumption that justifies this particular continuum model as a limit of finite economies.
} 
Lemma 5 Suppose that $x_{P}\left(\theta_{P}\right)$ as defined in (24) is weakly increasing and that $\phi_{P}^{*}$ is a solution to (20). Then, there exists some $p^{*}$ such that

$$
\phi_{P}^{*}\left(\theta_{P}\right)= \begin{cases}0 & \text { if } \theta_{P}<p^{*} \\ 1 & \text { if } \theta_{P} \geq p^{*} .\end{cases}
$$

Lemmas 4 and 5 show that, if the marginal distributions are such that the virtual surplus for each good is monotonic in type, the maximization of $(2)$ subject to $(\sqrt{\mathrm{IC}}),(\sqrt{\mathrm{IR}})$ and $(\mathrm{BB})$ reduces to a simple optimization problem in three variables, with some slight abuse of notations, (1) a flat user fee, $t^{*}$, for the public good; (2) a probability that the public good will be provided, $\phi_{G}^{*}$ (which may also be reinterpreted as the quantity of the public good); and (3) a fixed price, $p^{*}$, for the private good.

Hence, using Lemmas 4 and 5, we obtain the following simplified planning problem:

$$
\begin{array}{rl} 
& \max _{\left\{t, \phi_{G}, p\right\}} \phi_{G} \int_{\frac{t}{\phi_{G}}}^{\overline{\theta_{G}}} \theta_{G} d F_{G}\left(\theta_{G}\right)-K \phi_{G}+\int_{p}^{\overline{\theta_{P}}}\left(\theta_{P}-c\right) d F_{P}\left(\theta_{P}\right) \\
\text { s.t } 0 \leq t & t\left[1-F_{G}\left(\frac{t}{\phi_{G}}\right)\right]+(p-c)\left[1-F_{P}(p)\right]-K \phi_{G}, \\
0 \leq & \phi_{G} \leq 1 .
\end{array}
$$

Proposition 1 Suppose that $\mathrm{E} \theta_{G}>K$ and that $\underline{\theta_{G}}<K$. Then, in any optimal solution $\left(t^{*}, \phi_{G}^{*}, p^{*}\right)$ to (26), the following must be true:

1. $p^{*}>c$;

2. $\phi_{G}^{*}>0$;

3. $t^{*}>0$.

Proof. Let $\lambda, \mu$ and $\gamma$ respectively be the Lagrangian multiplier for the constraint (27) and the boundary constraints $\phi_{G} \geq 0$ and $\phi_{G} \leq 1$. The Kuhn-Tucker necessary conditions for an optimum are:

$$
\begin{aligned}
0 & =\int_{\frac{t}{\phi_{G}}}^{\overline{\theta_{G}}} \theta_{G} d F_{G}\left(\theta_{G}\right)+f_{G}\left(\frac{t}{\phi_{G}}\right)\left(\frac{t}{\phi_{G}}\right)^{2}-K+\lambda f_{G}\left(\frac{t}{\phi_{G}}\right)\left(\frac{t}{\phi_{G}}\right)^{2}-\lambda K+\mu-\gamma \\
\mu \phi_{G} & =0, \gamma\left(1-\phi_{G}\right)=0, \mu \geq 0, \gamma \geq 0 \\
0 & =-\frac{t}{\phi_{G}} f_{G}\left(\frac{t}{\phi_{G}}\right)+\lambda\left[1-F_{G}\left(\frac{t}{\phi_{G}}\right)-f_{G}\left(\frac{t}{\phi_{G}}\right) \frac{t}{\phi_{G}}\right] \\
0 & =-(p-c) f_{P}(p)+\lambda\left[\left(1-F_{P}(p)\right)-(p-c) f_{P}(p)\right] \\
0 & =\lambda\left\{t\left[1-F_{G}\left(\frac{t}{\phi_{G}}\right)\right]+(p-c)\left[1-F_{P}(p)\right]-K \phi_{G}\right\}, \lambda \geq 0
\end{aligned}
$$


PART 1: If $p^{*}<c$, then the first term on the right hand side in 32 is strictly positive and the second is weakly positive, implying that the condition cannot hold. Suppose that $p^{*}=c$. Then, from (32) either $\lambda=0$ or $1-F_{P}(c)=0$. Since the second condition is ruled out by the assumption that $c<\overline{\theta_{P}}$, the only possibility that remains is that $\lambda=0$. But if $\lambda=0$ at the optimal solution, then constraint (27) is not binding, which implies that $t^{*}, \phi_{G}^{*}$ must solve the following problem:

$$
\begin{array}{ll} 
& \max _{\left\{t, \phi_{G}\right\}} \phi_{G} \int_{\frac{t}{\phi_{G}}}^{\overline{\theta_{G}}} \theta_{G} d F_{G}\left(\theta_{G}\right)-K \phi_{G} \\
\text { s.t. } & 0 \leq \phi_{G} \leq 1 .
\end{array}
$$

We note that if $\phi_{G}^{*}>0$ in the solution, then the objective is monotonically decreasing in $t$ over $\left[\underline{\theta_{G}}, \overline{\theta_{G}}\right]$, thus if $\phi_{G}^{*}>0$, it must be that $t^{*}=\phi_{G}^{*} \underline{\theta_{G}}$. Thus, if $\phi_{G}^{*}>0$ it must maximize

$$
\phi_{G}\left[\int_{\underline{\theta_{G}}}^{\overline{\theta_{G}}} \theta_{G} d F_{G}\left(\theta_{G}\right)-K\right]=\phi_{G}\left[\mathrm{E} \theta_{G}-K\right] .
$$

By assumption the bracketed expression $\left[\mathrm{E} \theta_{G}-K\right]$ is strictly positive, so the solution must be $\phi_{G}^{*}=1$; and thus $t^{*}=\underline{\theta_{G}}$. But, substituting $p^{*}=c, t^{*}=\underline{\theta_{G}}$ and $\phi_{G}^{*}=1$ into the constraint (27) we see that

$$
t^{*}\left[1-F_{G}\left(\frac{t^{*}}{\phi_{G}^{*}}\right)\right]+\left(p^{*}-c\right)\left[1-F_{P}\left(p^{*}\right)\right]-K \phi_{G}^{*}=\underline{\theta_{G}}-K<0 .
$$

Hence, the resource constraint (27) is violated, which contradicts the assertion that $\lambda=0$. Thus we conclude that $p^{*}>c$ in any solution to 26 .

PART 2: Part 1 establishes that in the optimum $p^{*}>c$, thus if we have $\phi_{G}^{*}=0$, there must be a strict budget surplus (the tax collected from the private goods due to $p^{*}>c$ is unspent). It is easy to show that the social surplus can be improved by spending such budget surplus. To see this, fix $p^{*}, t^{*}=0, \phi_{G}^{*}=0$. But consider $\phi_{G}^{* \prime}$ given by

$$
\phi_{G}^{* \prime}=\frac{\left(p^{*}-c\right)\left[1-F_{P}\left(p^{*}\right)\right]}{K}>0
$$

By construction, constraint 27) is satisfied by the alternative simple mechanism $\left(t^{*}=0, \phi_{G}^{* \prime}, p^{*}\right)$. But the surplus under this alternative is

$$
\phi_{G}^{* \prime} \int_{0}^{\overline{\theta_{G}}} \theta_{G} d F_{G}\left(\theta_{G}\right)+\int_{p^{*}}^{\overline{\theta_{P}}}\left(\theta_{P}-c\right) d F_{P}\left(\theta_{P}\right)>\int_{p^{*}}^{\overline{\theta_{P}}}\left(\theta_{P}-c\right) d F_{P}\left(\theta_{P}\right) .
$$

Hence, $\left(t^{*}=0, \phi_{G}^{* \prime}, p^{*}\right)$ results in a strict increase in surplus relative to $\left(t^{*}=0, \phi_{G}^{*}=0, p^{*}\right)$. A contradiction. 
PART 3: This is obvious if $\underline{\theta_{G}}>0$, since $t^{*}=\phi_{G}^{*} \underline{\theta_{G}}$ would be non-distortionary. Suppose $t^{*}=0$ and $\underline{\theta_{G}} \leq 0$. Since the necessary condition $(31)$ must be satisfied, we have $\lambda\left[1-F_{G}(0)\right]=0$. This condition can only hold if $\lambda=0$. But we have shown in the proof of Part 1 that if $\lambda=0$, then $p^{*}=c$, which contradicts our conclusion in Part 1.

Thus, under some standard regularity conditions, the optimal separate provision mechanism is characterized by two prices - one for the public and one for the private good - and a probability to provide the public good. In particular, it shows that the only government intervention on the private goods under the optimal separate provision mechanism can be interpreted as a "unit tax" in the amount of $p^{*}-c$ and leave its provision solely to the "private sector." Hence, under the optimal separate provision mechanism, the private good can be provided in a completely decentralized manner via a competitive market (subject to a tax); and the unit tax on the private goods is used as a cross subsidy from the private good to the production of the public good. The logic is simply that the welfare cost of a small tax on the private good is second order, since the consumers who stop consuming the private good have valuations just marginally above the cost of production. What we find more interesting is that a public project with positive expected social benefits should always be undertaken with some positive probability. That is, there is a role for randomizations in the model. It is also interesting to observe that there should always be a strictly positive user fee for the public good. The logic for this result is similar to the argument for why there should be a positive tax on the private good, but runs counter the idea that excluding consumers when the marginal cost is zero is always bad.

The characterization of $\left(t^{*}, \phi_{G}^{*}, p^{*}\right)$ in Proposition 1 will be used as the comparison benchmark in the section in which we examine whether joint provision mechanisms can improve social welfare. In obtaining the above characterization, it is worth emphasizing again that we imposed only the restriction neither the access probabilities nor the fees for both the private and the public goods depends on the consumer's reported valuation of the other good, but did not impose any other restrictions were made on the set of admissible mechanisms. This is in contrast to the existing literature we reviewed in Section 2 where the desirability of public provision of private goods is typically established with some restriction on the available policy instruments or some kind of market imperfection when the government is not involved in the provision of the private goods. 


\section{$5 \quad$ Public Provision of Both Goods}

We have argued that the optimal separate provision mechanism characterized in Section 4 is consistent with an economy where the private good is traded on a competitive market (subject to a tax), and where the public good is provided by a government entity with resources coming from public good user fees and private good tax revenue. We will now consider a setup where the government is able to condition the provision probability and price for each of the two goods on the reported valuations of both goods. We interpret this as public provision of both goods since this is inconsistent with a world where the trading of the private good is done anonymously in the "private sector."

In general, a direct revelation mechanism can be represented as a quadruple $\left(\widetilde{\phi_{G}}, \widetilde{t}, \widetilde{\phi_{P}}, \widetilde{p}\right)$, where the difference with Section 4 is that all these functions are over the domain $\Theta_{G} \times \Theta_{P}$, whereas the corresponding objects in the separable case are functions of either $\Theta_{G}$ or $\Theta_{P}$. This leads to a multidimensional mechanism design problem, and there is no known methodology to characterize incentive compatibility in an analytically tractable way.

To get a tractable problem, we will proceed along the lines of McAfee et al. [25] and consider a class of simple mechanisms. Specifically, we will add a single instrument to the separate provision case, so that instead of considering mechanisms of the form $\left(t, \phi_{G}, p\right)$ as characterized in Proposition 1, we will consider mechanisms of the form $\left(t, \phi_{G}, p, \tau\right)$, where $\left(t, \phi_{G}, p\right)$ have the same interpretations as before, respectively as the user fee for the public good, the provision probability for the public good, and the price of the private good; but $\tau$ now is the fee charged for an agent who consumes both the public and the private good. If $\tau \neq t+p$, this requires that the government be actively involved in provision of the private good because such a scheme is feasible for the government only if it could monitor the consumers' purchases of the private goods.

While it is obviously a limitation that we are not able to characterize the constrained efficient mechanism for the full-blown mechanism design problem, the reader may note that, if we find that $\tau \neq t+p$ in the solution to our simplified problem, then it must be that the constrained efficient mechanism is also one in which the government takes an active part in the provision of the private good. Therefore, this suffices to answer the qualitative question we are interested in, namely whether public provision of a private good can be efficiency enhancing. 


\subsection{Some Preliminaries}

Consider a (simple pricing) mechanism on the form $\left(t, \phi_{G}, p, \tau\right)$. It is easy to see that a consumer will demand:

\section{Only the Public Good if:}

$$
\begin{aligned}
\phi_{G} \theta_{G}-t & \geq 0 \\
\phi_{G} \theta_{G}-t & \geq \theta_{P}-p \\
\phi_{G} \theta_{G}-t & \geq \phi_{G} \theta_{G}+\theta_{P}-\tau
\end{aligned}
$$

\section{Only the Private Good if:}

$$
\begin{aligned}
& \theta_{P}-p \geq 0 \\
& \theta_{P}-p \geq \phi_{G} \theta_{G}-t \\
& \theta_{P}-p \geq \phi_{G} \theta_{G}+\theta_{P}-\tau
\end{aligned}
$$

The Bundle if:

$$
\begin{aligned}
\phi_{G} \theta_{G}+\theta_{P}-\tau & \geq 0 \\
\phi_{G} \theta_{G}+\theta_{P}-\tau & \geq \phi_{G} \theta_{G}-t \\
\phi_{G} \theta_{G}+\theta_{P}-\tau & \geq \theta_{P}-p
\end{aligned}
$$

Which of the inequalities in (34), (35) and (36) are relevant depends on whether the bundle is cheaper or more expensive than the components. Figures 1 and 2 respectively represent the consumer's demand for the private and public goods as a function of her valuation types $\left(\theta_{G}, \theta_{P}\right)$ where for simplicity we multiplied her public valuation type by the provision probability $\phi_{G}$. The following two claims are obvious from the inspection of Figures 1 and 2 .

Claim 1 If $\tau \leq t+p$, the second inequality in (34) and (35) is implied by the other two inequalities. And,

- the proportion of agents purchasing the public good only is

$$
\int_{\frac{t}{\phi_{G}}}^{\overline{\theta_{G}}} \int_{\underline{\theta_{P}}}^{\tau-t} f\left(\theta_{G}, \theta_{P}\right) d \theta_{P} d \theta_{G}
$$




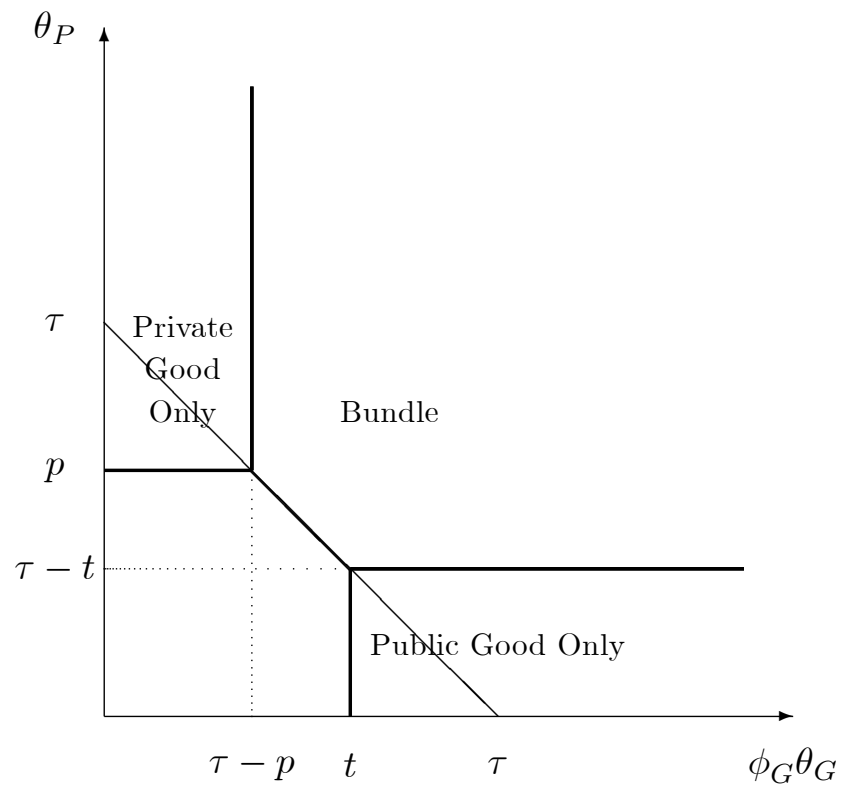

Figure 1: Consumers' Demand for the Case $\tau \leq t+p$.

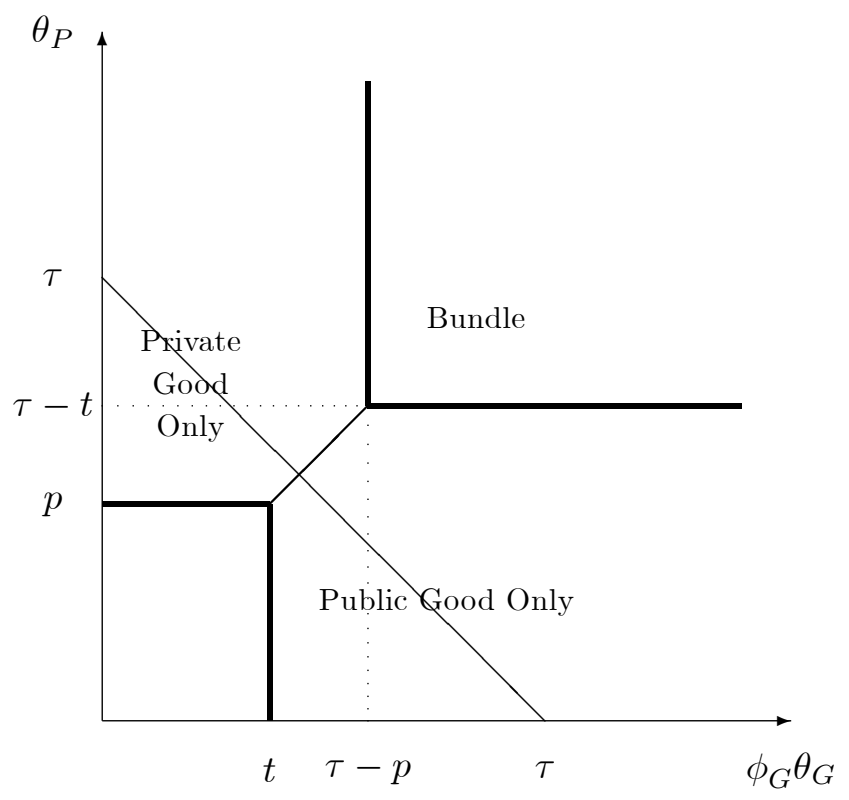

Figure 2: Consumers' Demand for the Case $\tau>t+p$. 
- the proportion of agents purchasing the private good only is

$$
\int_{p}^{\overline{\theta_{P}}} \int_{\underline{\theta_{G}}}^{\frac{\tau-p}{\phi_{G}}} f\left(\theta_{G}, \theta_{P}\right) d \theta_{G} d \theta_{P}
$$

- the proportion of agents purchasing the bundle is

$$
\int_{\frac{\tau-p}{\phi_{G}}}^{\frac{t}{\phi_{G}}} \int_{\tau-\phi_{G} \theta_{G}}^{\overline{\theta_{P}}} f\left(\theta_{G}, \theta_{P}\right) d \theta_{P} d \theta_{G}+\int_{\frac{t}{\phi_{G}}}^{\overline{\theta_{G}}} \int_{\tau-t}^{\overline{\theta_{P}}} f\left(\theta_{G}, \theta_{P}\right) d \theta_{P} d \theta_{G}
$$

Claim 2 If $\tau \geq t+p$, the first inequality in (36) is implied by the other two inequalities. And,

- the proportion of agents purchasing the public good only is

$$
\int_{\frac{t}{\phi_{G}}}^{\frac{\tau-p}{\phi_{G}}} \int_{\underline{\theta_{P}}}^{\phi_{G} \theta_{G}+p-t} f\left(\theta_{G}, \theta_{P}\right) d \theta_{P} d \theta_{G}+\int_{\frac{\tau-p}{\phi_{G}}}^{\overline{\theta_{G}}} \int_{\underline{\theta_{P}}}^{\tau-t} f\left(\theta_{G}, \theta_{P}\right) d \theta_{P} d \theta_{G}
$$

- the proportion of agents purchasing the private good only is

$$
\int_{p}^{\tau-t} \int_{\underline{\theta}}^{\frac{\theta_{P}+t-p}{\phi_{G}}} f\left(\theta_{G}, \theta_{P}\right) d \theta_{G} d \theta_{P}+\int_{\tau-t}^{\overline{\theta_{P}}} \int_{\underline{\theta}}^{\frac{\tau-p}{\phi_{G}}} f\left(\theta_{G}, \theta_{P}\right) d \theta_{G} d \theta_{P}
$$

- the proportion of agents purchasing the bundle is

$$
\int_{\frac{\tau-p}{\phi_{G}}}^{\overline{\theta_{G}}} \int_{\tau-t}^{\overline{\theta_{P}}} f\left(\theta_{G}, \theta_{P}\right) d \theta_{P} d \theta_{G}
$$

Define by $G_{1}\left(t, p, \tau ; \phi_{G}\right)$ the budget surplus (when positive) given a mechanism $\left(t, \phi_{G}, p, \tau\right)$ under case one when $\tau \leq t+p$. Using Claim 1, it can be written as:

$$
\begin{aligned}
G_{1}\left(t, p, \tau ; \phi_{G}\right)= & t\left[\int_{\frac{t}{\phi_{G}}}^{\overline{\theta_{G}}} \int_{\underline{\theta_{P}}}^{\tau-t} f\left(\theta_{G}, \theta_{P}\right) d \theta_{P} d \theta_{G}\right]+(p-c)\left[\int_{p}^{\overline{\theta_{P}}} \int_{\underline{\theta}}^{\frac{\tau-p}{\phi_{G}}} f\left(\theta_{G}, \theta_{P}\right) d \theta_{G} d \theta_{P}\right] \\
& +(\tau-c)\left[\int_{\frac{\tau-p}{\phi_{G}}}^{\overline{\frac{t}{\phi_{G}}}} \int_{\tau-\phi_{G} \theta_{G}}^{\overline{\theta_{P}}} f\left(\theta_{G}, \theta_{P}\right) d \theta_{P} d \theta_{G}+\int_{\frac{t}{\bar{\phi}_{G}}}^{\overline{\theta_{G}}} \int_{\tau-t}^{\overline{\theta_{P}}} f\left(\theta_{G}, \theta_{P}\right) d \theta_{P} d \theta_{G}\right]-K \phi_{G},
\end{aligned}
$$

Symmetrically, we let $G_{2}\left(t, p, \tau ; \phi_{G}\right)$ denote the budget surplus given a mechanism $\left(t, \phi_{G}, p, \tau\right)$ where $\tau \geq t+p$, which using Claim 2 can be written as:

$$
\begin{aligned}
G_{2}\left(t, p, \tau ; \phi_{G}\right)= & t\left[\int_{\frac{t}{\phi_{G}}}^{\frac{\tau-p}{\phi_{G}}} \int_{\underline{\theta_{P}}}^{\phi_{G} \theta_{G}+p-t} f\left(\theta_{G}, \theta_{P}\right) d \theta_{P} d \theta_{G}+\int_{\frac{\tau-p}{\phi_{G}}}^{\overline{\theta_{G}}} \int_{\underline{\theta}_{P}}^{p} f\left(\theta_{G}, \theta_{P}\right) d \theta_{P} d \theta_{G}\right] \\
& +(p-c)\left[\int_{p}^{\tau-t} \int_{\underline{\theta}}^{\frac{\theta_{P}+t-p}{\phi_{G}}} f\left(\theta_{G}, \theta_{P}\right) d \theta_{G} d \theta_{P}+\int_{\tau-t}^{\overline{\theta_{P}}} \int_{\underline{\theta}}^{\frac{\tau-p}{\phi_{G}}} f\left(\theta_{G}, \theta_{P}\right) d \theta_{G} d \theta_{P}\right] \\
& +(\tau-c)\left[\int_{\frac{\tau-p}{\phi_{G}}}^{\overline{\theta_{G}}} \int_{\tau-t}^{\overline{\theta_{P}}} f\left(\theta_{G}, \theta_{P}\right) d \theta_{P} d \theta_{G}\right]-K \phi_{G} .
\end{aligned}
$$


Next, let $S_{1}\left(t, p, \tau ; \phi_{G}\right)$ denote the social surplus associated with $\left(t, \phi_{G}, p, \tau\right)$ in the case when $\tau \leq t+p$,

$$
\begin{aligned}
S_{1}\left(t, p, \tau ; \phi_{G}\right)= & \int_{\overline{\frac{t}{\phi_{G}}}}^{\overline{\theta_{G}}} \int_{\theta_{P}}^{\tau-t} \phi_{G} \theta_{G} f\left(\theta_{G}, \theta_{P}\right) d \theta_{P} d \theta_{G}+\int_{p}^{\overline{\theta_{P}}} \int_{\underline{\theta_{G}}}^{\frac{\tau-p}{\phi_{G}}}\left(\theta_{P}-c\right) f\left(\theta_{G}, \theta_{P}\right) d \theta_{G} d \theta_{P} \\
& +\int_{\frac{\tau-p}{\phi_{G}}}^{\overline{\frac{t}{\phi_{G}}}} \int_{\tau-\phi_{G} \theta_{G}}^{\overline{\theta_{P}}}\left(\phi_{G} \theta_{G}+\theta_{P}-c\right) f\left(\theta_{G}, \theta_{P}\right) d \theta_{P} d \theta_{G} \\
& +\int_{\frac{t}{\phi_{G}}}^{\overline{\theta_{G}}} \int_{\tau-t}^{\overline{\theta_{P}}}\left(\phi_{G} \theta_{G}+\theta_{P}-c\right) f\left(\theta_{G}, \theta_{P}\right) d \theta_{P} d \theta_{G}-K \phi_{G} .
\end{aligned}
$$

and let $S_{2}\left(t, p, \tau ; \phi_{G}\right)$ be the social surplus associated with $\left(t, \phi_{G}, p, \tau\right)$ in the case when $\tau \geq t+p$,

$$
\begin{aligned}
S_{2}\left(t, p, \tau ; \phi_{G}\right)= & \int_{\frac{t}{\phi_{G}}}^{\frac{\tau-p}{\phi_{G}}} \int_{\underline{\theta_{P}}}^{\phi_{G} \theta_{G}+p-t} \phi_{G} \theta_{G} f\left(\theta_{G}, \theta_{P}\right) d \theta_{P} d \theta_{G}+\int_{\frac{\tau-p}{\phi_{G}}}^{\overline{\theta_{G}}} \int_{\underline{\theta_{P}}}^{\tau-t} \phi_{G} \theta_{G} f\left(\theta_{G}, \theta_{P}\right) d \theta_{P} d \theta_{G} \\
& +\int_{p}^{\tau-t} \int_{\underline{\theta_{P}}}^{\frac{\theta_{P}+t-p}{\phi_{G}}}\left(\theta_{P}-c\right) f\left(\theta_{G}, \theta_{P}\right) d \theta_{G} d \theta_{P}+\int_{\tau-t}^{\theta_{P}} \int_{\underline{\theta^{\prime}}}^{\frac{\tau-p}{\phi_{G}}}\left(\theta_{P}-c\right) f\left(\theta_{G}, \theta_{P}\right) d \theta_{G} d \theta_{P} \\
& +\int_{\frac{\tau-p}{\phi_{G}}}^{\overline{\theta_{G}}} \int_{\tau-t}^{\overline{\theta_{P}}}\left(\phi_{G} \theta_{G}+\theta_{P}-c\right) f\left(\theta_{G}, \theta_{P}\right) d \theta_{P} d \theta_{G}-K \phi_{G} .
\end{aligned}
$$

For notational brevity we will let $z=(t, p, \tau)$ and $z^{*}=\left(t^{*}, p^{*}, t^{*}+p^{*}\right)$, where $\left(t^{*}, \phi_{G}^{*}, p^{*}\right)$ is an optimal solution to the separate provision problem (26) in Section 4 . Now we define two auxiliary problems. First consider the problem of:

$$
\begin{array}{ll} 
& \max _{(t, p, \tau)} S_{1}\left(t, p, \tau ; \phi_{G}^{*}\right) \\
\text { s.t. } & G_{1}\left(t, p, \tau ; \phi_{G}^{*}\right) \geq 0 \\
& t+p-\tau \geq 0 .
\end{array}
$$

Problem (41) gives the best simple pricing policy in the form of $\left(t, \phi_{G}, p, \tau\right)$ under the restriction that $\phi_{G}=\phi_{G}^{*}$ and $\tau \leq t+p$; that is, the public good provision probability is fixed at the level as in the optimal separate provision mechanism characterized in Proposition 1, and the bundle is restricted to be no more expensive than separate purchase of its components. Problem (41) will tell us the maximal attainable social surplus when one deviates from the the optimal separable provision mechanism among a particular set of perturbations (namely, charging the bundle of public and private goods a lower price than the sum of its components), while respecting the budget balance constraint. Notice that the separate provision mechanism prices $z^{*}=\left(t^{*}, p^{*}, t^{*}+p^{*}\right)$ is in the constraint set of Problem 41). 
Symmetrically, consider the problem of:

$$
\begin{array}{ll} 
& \max _{(t, p, \tau)} S_{2}\left(t, p, \tau ; \phi_{G}^{*}\right) \\
\text { s.t. } & G_{2}\left(t, p, \tau ; \phi_{G}^{*}\right) \geq 0 \\
& \tau-t-p \geq 0 .
\end{array}
$$

Problem (42) gives the best simple pricing policy in the form of $\left(t, \phi_{G}, p, \tau\right)$ under the restriction that $\phi_{G}=\phi_{G}^{*}$ and $\tau \geq t+p$. That is, the public good provision probability is fixed at the level as in the optimal separate provision mechanism characterized in Proposition 1, and the bundle is restricted to be no cheaper than separate purchase of its components. Problem (42) will tell us the maximal attainable social surplus when one deviates from the the optimal separable provision mechanism among another particular set of perturbations (namely, charging the bundle of public and private goods a higher price than the sum of its components), while respecting the budget balance constraint. Notice again that the separate provision mechanism prices $z^{*}=\left(t^{*}, p^{*}, t^{*}+p^{*}\right)$ is in the constraint set of Problem 42.

If there is no potential improvement of joint provision mechanism over the optimal separate provision mechanism we characterized in Proposition 1, a necessary condition is that $\left(t^{*}, p^{*}, \tau=t^{*}+p^{*}\right)$ must solve both Problems 41) and (42).

\subsection{The Main Result}

Now we show our main result, which is a sufficient condition under which $\left(t^{*}, p^{*}, \tau=t^{*}+p^{*}\right)$ cannot simultaneously solve both Problems (41) and (42). As a result, when the identified condition is satisfied, there must exist a simple pricing joint provision mechanism that improves social welfare upon the optimal separate provision mechanisms.

To this end, we first introduce a few notations. Differentiating (37) and evaluating at $z=z^{*}=$ 
$\left(t^{*}, p^{*}, t^{*}+p^{*}\right)$, we find that:16

$$
\begin{aligned}
\frac{\partial G_{1}\left(z^{*} ; \phi_{G}^{*}\right)}{\partial t}= & \int_{\frac{t^{*}}{\phi_{G}^{*}}}^{\overline{\theta_{G}}}\left\{F_{P}\left(p^{*} \mid \theta_{G}\right)+\left(p^{*}-c\right) f_{P}\left(p^{*} \mid \theta_{G}\right)\right\} f_{G}\left(\theta_{G}\right) d \theta_{G} \\
& -\frac{t^{*}}{\phi_{G}^{*}} F_{P}\left(p^{*} \mid \frac{t^{*}}{\phi_{G}^{*}}\right) f_{G}\left(\frac{t^{*}}{\phi_{G}^{*}}\right) \\
\frac{\partial G_{1}\left(z^{*} ; \phi_{G}^{*}\right)}{\partial p}= & \int_{\frac{\theta_{G}}{\phi_{G}^{*}}}^{\frac{t^{*}}{t^{*}}}\left[\left\{\left(1-F_{P}\left(p^{*} \mid \theta_{G}\right)\right)-\left(p^{*}-c\right) f_{P}\left(p^{*} \mid \theta_{G}\right)\right\}\right] f_{G}\left(\theta_{G}\right) d \theta_{G} \\
& +\frac{t^{*}}{\phi_{G}^{*}}\left[1-F_{P}\left(p^{*} \mid \frac{t^{*}}{\phi_{G}^{*}}\right)\right] f_{G}\left(\frac{t^{*}}{\phi_{G}^{*}}\right) \\
\frac{\partial G_{1}\left(z^{*} ; \phi_{G}^{*}\right)}{\partial \tau}= & \int_{\frac{t^{*}}{\phi_{G}^{*}}}^{\frac{\theta_{G}}{\partial}}\left\{\left(1-F_{P}\left(p^{*} \mid \theta_{G}\right)\right)-\left(p^{*}-c\right) f_{P}\left(p^{*} \mid \theta_{G}\right)\right\} f_{G}\left(\theta_{G}\right) d \theta_{G} \\
& -\frac{t^{*}}{\phi_{G}^{*}}\left[1-F_{P}\left(p^{*} \mid \frac{t^{*}}{\phi_{G}^{*}}\right)\right] f_{G}\left(\frac{t^{*}}{\phi_{G}^{*}}\right)
\end{aligned}
$$

These expressions inform us about the effect on the budget when one slightly perturbs the relevant prices $t, p$ and $\tau$.

Likewise, if we differentiate $S_{1}(\cdot)$ in $(39)$ and evaluate at $z=z^{*}$, we obtain:

$$
\begin{aligned}
& \frac{\partial S_{1}\left(z^{*} ; \phi_{G}^{*}\right)}{\partial t}=\int_{\frac{t^{*}}{\phi_{G}^{*}}}^{\overline{\theta_{G}}}\left(p^{*}-c\right) f_{P}\left(p^{*} \mid \theta_{G}\right) f_{G}\left(\theta_{G}\right) d \theta_{G}-\frac{t^{*}}{\phi_{G}^{*}} F_{P}\left(p^{*} \mid \frac{t^{*}}{\phi_{G}^{*}}\right) f_{G}\left(\frac{t^{*}}{\phi_{G}^{*}}\right) \\
& \frac{\partial S_{1}\left(z^{*} ; \phi_{G}^{*}\right)}{\partial p}=-\int_{\theta_{G}}^{\frac{t_{G}^{*}}{\phi_{G}^{*}}}\left(p^{*}-c\right) f_{P}\left(p^{*} \mid \theta_{G}\right) f_{G}\left(\theta_{G}\right) d \theta_{G}+\frac{t^{*}}{\phi_{G}^{*}}\left[1-F_{P}\left(p^{*} \mid \frac{t^{*}}{\phi_{G}^{*}}\right)\right] f_{G}\left(\frac{t^{*}}{\phi_{G}^{*}}\right) \\
& \frac{\partial S_{1}\left(z^{*} ; \phi_{G}^{*}\right)}{\partial \tau}=-\int_{\frac{t^{*}}{\bar{\theta}_{G}^{*}}}^{\bar{\theta}_{G}}\left(p^{*}-c\right) f_{P}\left(p^{*} \mid \theta_{G}\right) f_{G}\left(\theta_{G}\right) d \theta_{G}-\frac{t^{*}}{\phi_{G}^{*}}\left[1-F_{P}\left(p^{*} \mid \frac{t^{*}}{\phi_{G}^{*}}\right)\right] f_{G}\left(\frac{t^{*}}{\phi_{G}^{*}}\right) .
\end{aligned}
$$

We can also write out explicitly the gradients for $S_{2}$ and $G_{2}$, which we omit here.

Remark. The reader may notice that expression $\partial G_{1}\left(z^{*} ; \phi_{G}^{*}\right) / \partial p$ in $43 \mathrm{~b}$ corresponds directly to the expression in Proposition 1 of McAfee et al. (1989). Expression (43a) can also be written in that form by reversing the roles of $\theta_{G}$ and $\theta_{P}$. This close correspondence with McAfee et al. (1989) is not a coincidence. The derivatives reported above can be thought of as the effect on profits given a marginal increase in $t, p$ and $\tau$ respectively, which is exactly what McAfee et al. (1989) was analyzing. In their case, going from $43 \mathrm{~b}$ to their main result is relatively straightforward since they asked for a direction where the mixed bundling mechanism increases profits relative to separate pricing. In particular, $\left(t^{*}, p^{*}\right)$ in their case were chosen to solve a monopolist profit maximization problem under separate pricing, thus $p^{*}$, for example, must satisfy the first order optimality condition $\left[1-F_{P}\left(p^{*}\right)\right]-\left(p^{*}-c\right) f_{P}\left(p^{*}\right)=0$. Hence, the first term in (43b), if $\theta_{G}$ and

\footnotetext{
${ }^{16}$ The details of the derivations for 43 and 44 are available in an Appendix from the authors' websites.
} 
$\theta_{P}$ are stochastically independent, becomes:

$$
\begin{aligned}
& \int_{\underline{\theta_{G}}}^{\frac{t^{*}}{\phi_{G}^{*}}}\left\{\left[1-F_{P}\left(p^{*} \mid \theta_{G}\right)\right]-\left(p^{*}-c\right) f_{P}\left(p^{*} \mid \theta_{G}\right)\right\} f_{G}\left(\theta_{G}\right) d \theta_{G} \\
= & \int_{\underline{\theta_{G}}}^{\frac{t^{*}}{\phi_{G}^{*}}}\left\{\left[1-F_{P}\left(p^{*}\right)\right]-\left(p^{*}-c\right) f_{P}\left(p^{*}\right)\right\} f_{G}\left(\theta_{G}\right),
\end{aligned}
$$

which is equal to zero from the optimality condition of $p^{*}$ in their problem. Thus it follows immediately from (43b) that a small increase in the price of the private good (or a small decrease in the price from the bundle) would increase the profits in the case of stochastic independence. It can also be verified that, by rewriting $\partial G_{1}\left(z^{*} ; \phi_{G}^{*}\right) / \partial t$ (to be explicit about the optimality condition for $t^{*}$ for the monopolist profit-maximization problem), that a small increase in the price of the public good also increases profits if $\theta_{G}$ and $\theta_{P}$ are stochastically independent.

Our problem differs from McAfee et al. (1989) in two respects. First of all, our goal is to demonstrate that bundling can increase social welfare rather than profits. Secondly, because $\left(t^{*}, p^{*}\right)$ in our problem, as characterized in Proposition 1, are not chosen to maximize profits, we cannot use the first order conditions from the optimal separable provision mechanism in the same way as McAfee et al. (1989).

Our first preliminary result is that in fact, evaluated at an optimal separate provision mechanism prices $\left(t^{*}, p^{*}, \tau=t^{*}+p^{*}\right)$, the partial derivatives of $G_{1}$ and $G_{2}$ are the same, and the partial derivatives of $S_{1}$ and $S_{2}$ also coincide. Letting $D G_{i}\left(z ; \phi_{G}\right)$ and $D S_{i}\left(z ; \phi_{G}\right)$ denote the gradient vectors for $i=1,2$, we thus have that;

Lemma $6 D G_{1}\left(z^{*} ; \phi_{G}^{*}\right)=D G_{2}\left(z^{*} ; \phi_{G}^{*}\right)$ and $D S_{1}\left(z^{*} ; \phi_{G}^{*}\right)=D S_{2}\left(z^{*} ; \phi_{G}^{*}\right)$

Now we establish a useful lemma:

Lemma 7 Let $\lambda^{*}$ be the multiplier on constraint (27) corresponding to the solution $\left(\phi_{G}^{*}, t^{*}, p^{*}\right)$ of problem (26). Also, let $\lambda_{i}$ be the multiplier on the resource constraint $G_{i}\left(t, p, \tau ; \phi_{G}^{*}\right)$ for $i=1,2$ in problem (41) and (42). Then,

1. $\lambda_{1}=\lambda^{*}$ if $z^{*}$ solves problem $(41)$;

2. $\lambda_{2}=\lambda^{*}$ if $z^{*}$ solves problem (42). 
Proof. First consider (41). If $z^{*}$ solves the problem, the Kuhn-Tucker necessary conditions for a solution must be fulfilled at $z^{*}$. Hence, there must exist $\lambda_{1}>0$ and $\mu_{1} \geq 0$ such that

$$
\begin{aligned}
& \frac{\partial S_{1}\left(z^{*} ; \phi_{G}^{*}\right)}{\partial t}+\lambda_{1} \frac{\partial G_{1}\left(z^{*} ; \phi_{G}^{*}\right)}{\partial t}+\mu_{1}=0 \\
& \frac{\partial S_{1}\left(z^{*} ; \phi_{G}^{*}\right)}{\partial p}+\lambda_{1} \frac{\partial G_{1}\left(z^{*} ; \phi_{G}^{*}\right)}{\partial p}+\mu_{1}=0 \\
& \frac{\partial S_{1}\left(z^{*} ; \phi_{G}^{*}\right)}{\partial \tau}+\lambda_{1} \frac{\partial G_{1}\left(z^{*} ; \phi_{G}^{*}\right)}{\partial \tau}-\mu_{1}=0 \\
& \mu_{1}(t+p-\tau)=0, \quad \mu_{1} \geq 0
\end{aligned}
$$

Using the expressions for the partial derivatives in (43) and 44), it is easy to check that:

$$
\begin{aligned}
\frac{\partial S_{1}\left(z^{*} ; \phi_{G}^{*}\right)}{\partial t}+\frac{\partial S_{1}\left(z^{*} ; \phi_{G}^{*}\right)}{\partial \tau} & =-\frac{t^{*}}{\phi_{G}^{*}} f_{G}\left(\frac{t^{*}}{\phi_{G}^{*}}\right) \\
\frac{\partial G_{1}\left(z^{*} ; \phi_{G}^{*}\right)}{\partial t}+\frac{\partial G_{1}\left(z^{*} ; \phi_{G}^{*}\right)}{\partial \tau} & =\int_{\frac{t^{*}}{\phi_{G}^{*}}}^{\theta_{G}} f_{G}\left(\theta_{G}\right) d \theta_{G}-\frac{t^{*}}{\phi_{G}^{*}} f_{G}\left(\frac{t^{*}}{\phi_{G}^{*}}\right) \\
& =\left[1-F_{G}\left(\frac{t^{*}}{\phi_{G}^{*}}\right)\right]-\frac{t^{*}}{\phi_{G}^{*}} f_{G}\left(\frac{t^{*}}{\phi_{G}^{*}}\right) .
\end{aligned}
$$

Combining 46a and (46c), and using (47), we have that

$$
-\frac{t^{*}}{\phi_{G}^{*}} f_{G}\left(\frac{t^{*}}{\phi_{G}^{*}}\right)+\lambda_{1}\left\{\left[1-F_{G}\left(\frac{t^{*}}{\phi_{G}^{*}}\right)\right]-\frac{t^{*}}{\phi_{G}^{*}} f_{G}\left(\frac{t^{*}}{\phi_{G}^{*}}\right)\right\}=0 .
$$

This condition is the same as 31, the first order condition to the problem when the goods are sold separately. It follows that $\lambda_{1}=\lambda^{*}$, since otherwise (48) will be violated. This proves the first part.

For the second part, we note that the Kuhn-Tucker conditions for Problem (42) are

$$
\begin{aligned}
\frac{\partial S_{2}\left(z^{*} ; \phi_{G}^{*}\right)}{\partial t}+\lambda_{2} \frac{\partial G_{2}\left(z^{*} ; \phi_{G}^{*}\right)}{\partial t}-\mu_{2} & =\frac{\partial S_{1}\left(z^{*} ; \phi_{G}^{*}\right)}{\partial t}+\lambda_{2} \frac{\partial G_{1}\left(z^{*} ; \phi_{G}^{*}\right)}{\partial t}-\mu_{2}=0 \\
\frac{\partial S_{2}\left(z^{*} ; \phi_{G}^{*}\right)}{\partial p}+\lambda_{2} \frac{\partial G_{2}\left(z^{*} ; \phi_{G}^{*}\right)}{\partial p}-\mu_{2} & =\frac{\partial S_{1}\left(z^{*} ; \phi_{G}^{*}\right)}{\partial p}+\lambda_{2} \frac{\partial G_{1}\left(z^{*} ; \phi_{G}^{*}\right)}{\partial p}-\mu_{2}=0 \\
\frac{\partial S_{2}\left(z^{*} ; \phi_{G}^{*}\right)}{\partial \tau}+\lambda_{2} \frac{\partial G_{2}\left(z^{*} ; \phi_{G}^{*}\right)}{\partial \tau}+\mu_{2} & =\frac{\partial S_{1}\left(z^{*} ; \phi_{G}^{*}\right)}{\partial \tau}+\lambda_{2} \frac{\partial G_{1}\left(z^{*} ; \phi_{G}^{*}\right)}{\partial \tau}+\mu_{2}=0 \\
\mu_{2}(t+p-\tau) & =0, \mu_{2} \geq 0
\end{aligned}
$$

where the first equality in each line follows from Lemma 6. The same argument applies.

Together, Lemmas 6 and 7 makes the Kuhn-Tucker conditions for problem 41] comparable with those of problem 42. Now our main result about the sufficient condition for the welfare improvement potential of public provision of private goods over the optimal separate provision mechanism follows: 
Proposition 2 Let $\lambda^{*}$ be the multiplier on constraint (27) corresponding to the solution $\left(\phi_{G}^{*}, t^{*}, p^{*}\right)$ of problem (26). Then, there exists a feasible simple pricing policy $(t, p, \tau)$ that generates a higher social surplus than the optimal separate provision mechanism whenever

$$
D S_{1}\left(z^{*} ; \phi_{G}^{*}\right)+\lambda^{*} D G_{1}\left(z^{*} ; \phi_{G}^{*}\right) \neq 0
$$

Proof. From Lemma 7, we know that if $z^{*}$ solves both problems 41) and 42, the multiplier in each problem must be given by $\lambda^{*}$. Thus if $z^{*}$ is the best simple pricing policy for problem (41), then

$$
\begin{aligned}
\frac{\partial S_{1}\left(z^{*} ; \phi_{G}^{*}\right)}{\partial t}+\lambda^{*} \frac{\partial G_{1}\left(z^{*} ; \phi_{G}^{*}\right)}{\partial t}+\mu_{1} & =0 \\
\frac{\partial S_{1}\left(z^{*} ; \phi_{G}^{*}\right)}{\partial p}+\lambda^{*} \frac{\partial G_{1}\left(z^{*} ; \phi_{G}^{*}\right)}{\partial p}+\mu_{1} & =0 \\
\frac{\partial S_{1}\left(z^{*} ; \phi_{G}^{*}\right)}{\partial \tau}+\lambda^{*} \frac{\partial G_{1}\left(z^{*} ; \phi_{G}^{*}\right)}{\partial \tau}-\mu_{1} & =0 \\
\mu_{1}(t+p-\tau)=0, & \quad \mu_{1} \geq 0 .
\end{aligned}
$$

Similarly if $z^{*}$ is the best simple pricing policy for problem $(42)$, then by using Lemma 6, we have

$$
\begin{aligned}
\frac{\partial S_{1}\left(z^{*} ; \phi_{G}^{*}\right)}{\partial t}+\lambda^{*} \frac{\partial G_{1}\left(z^{*} ; \phi_{G}^{*}\right)}{\partial t}-\mu_{2} & =0 \\
\frac{\partial S_{1}\left(z^{*} ; \phi_{G}^{*}\right)}{\partial p}+\lambda^{*} \frac{\partial G_{1}\left(z^{*} ; \phi_{G}^{*}\right)}{\partial p}-\mu_{2} & =0 \\
\frac{\partial S_{1}\left(z^{*} ; \phi_{G}^{*}\right)}{\partial \tau}+\lambda^{*} \frac{\partial G_{1}\left(z^{*} ; \phi_{G}^{*}\right)}{\partial \tau}+\mu_{2} & =0 \\
\mu_{2}(t+p-\tau)=0, & \quad \mu_{2} \geq 0
\end{aligned}
$$

Assume that $\mu_{1}>0$. Then, 50 implies that $\frac{\partial S_{1}\left(z^{*} ; \phi_{G}^{*}\right)}{\partial t}+\lambda^{*} \frac{\partial G_{1}\left(z^{*} ; \phi_{G}^{*}\right)}{\partial t}<0$, which makes it impossible to find $\mu_{2} \geq 0$ such that 51 holds. Symmetrically, if $\mu_{2}>0$, then $\frac{\partial S_{1}\left(z^{*} ; \phi_{G}^{*}\right)}{\partial t}+$ $\lambda^{*} \frac{\partial G_{1}\left(z^{*} ; \phi_{G}^{*}\right)}{\partial t}>0$, which makes it impossible to find $\mu_{1} \geq 0$ such that 50 holds. Since $z^{*}$ must solve both (41) and 42 for there to be no improvement we conclude that $\mu_{1}=\mu_{2}=0$, or else there is some $z$ better than $z^{*}$. The claim follows.

Because the simple pricing joint provision mechanism is a subset of all feasible joint provision mechanisms, an immediate implication of Proposition 2 is that whenever condition 49) is satisfied, the optimal joint provision mechanism must generate higher social welfare than the optimal separate provision mechanism. 


\subsection{Stochastic Independence}

Now we use Proposition 2 above to examine the case where $\theta_{G}$ and $\theta_{P}$ are stochastically independent. In this case we have that there is indeed always an improvement over the optimal separate provision policy.

Proposition 3 Suppose that $\theta_{G}$ and $\theta_{P}$ are stochastically independent. Then

$$
\frac{\partial S_{1}\left(z^{*} ; \phi_{G}^{*}\right)}{\partial p}+\lambda^{*} \frac{\partial G_{1}\left(z^{*} ; \phi_{G}^{*}\right)}{\partial p}>0
$$

Proof. When $f_{P}\left(\theta_{P} \mid \theta_{G}\right)=f_{P}\left(\theta_{P}\right)$ for all $\theta_{P}$ we have that

$$
\begin{aligned}
\frac{\partial G_{1}\left(z^{*} ; \phi_{G}^{*}\right)}{\partial p} & =\left[1-F_{P}\left(p^{*}\right)-\left(p^{*}-c\right) f_{P}\left(p^{*}\right)\right] F_{G}\left(\frac{t^{*}}{\phi_{G}^{*}}\right)+\frac{t^{*}}{\phi_{G}^{*}}\left[1-F_{P}\left(p^{*}\right)\right] f_{G}\left(\frac{t^{*}}{\phi_{G}^{*}}\right) \\
& =\frac{\left(p^{*}-c\right) f_{P}\left(p^{*}\right)}{\lambda^{*}} F_{G}\left(\frac{t^{*}}{\phi_{G}^{*}}\right)+\frac{t^{*}}{\phi_{G}^{*}}\left[1-F_{P}\left(p^{*}\right)\right] f_{G}\left(\frac{t^{*}}{\phi_{G}^{*}}\right)
\end{aligned}
$$

where the second equality uses (32), the first order condition for $p^{*}$ in the separable case. Next,

$$
\frac{\partial S_{1}\left(z^{*} ; \phi_{G}^{*}\right)}{\partial p}=-\left(p^{*}-c\right) f_{P}\left(p^{*}\right) F_{G}\left(\frac{t^{*}}{\phi_{G}^{*}}\right)+\frac{t^{*}}{\phi_{G}^{*}}\left[1-F_{P}\left(p^{*}\right)\right] f_{G}\left(\frac{t^{*}}{\phi_{G}^{*}}\right)
$$

Hence,

$$
\begin{aligned}
\frac{\partial S_{1}\left(z^{*} ; \phi_{G}^{*}\right)}{\partial p}+\lambda^{*} \frac{\partial G_{1}\left(z^{*} ; \phi_{G}^{*}\right)}{\partial p}= & -\left(p^{*}-c\right) f_{P}\left(p^{*}\right) F_{G}\left(\frac{t^{*}}{\phi_{G}^{*}}\right)+\frac{t^{*}}{\phi_{G}^{*}}\left[1-F_{P}\left(p^{*}\right)\right] f_{G}\left(\frac{t^{*}}{\phi_{G}^{*}}\right) \\
& +\lambda^{*}\left\{\frac{\left(p^{*}-c\right) f_{P}\left(p^{*}\right)}{\lambda^{*}} F_{G}\left(\frac{t^{*}}{\phi_{G}^{*}}\right)+\frac{t^{*}}{\phi_{G}^{*}}\left[1-F_{P}\left(p^{*}\right)\right] f_{G}\left(\frac{t^{*}}{\phi_{G}^{*}}\right)\right\} \\
= & \left(1+\lambda^{*}\right) \frac{t^{*}}{\phi_{G}^{*}}\left[1-F_{P}\left(p^{*}\right)\right] f_{G}\left(\frac{t^{*}}{\phi_{G}^{*}}\right)>0 .
\end{aligned}
$$

Proposition 3 establishes that when the valuations for the private and public goods are stochastically independent, there is a welfare improving role for the public provision of private goods over the optimal separate provision mechanism we characterized in Proposition 1. It also implies, by continuity, that there exists an open set of joint distribution functions for $\theta_{G}$ and $\theta_{P}$ for which public provision of private goods can be preferred from an efficiency viewpoint ${ }^{17}$

\footnotetext{
${ }^{17}$ We conjecture is that condition 49 is satisfied generically in the sense that it holds for almost all joint distribution functions.
} 


\section{Conclusion}

This paper shows that public provision of private goods may be justified on pure efficiency grounds in an environment where individuals consume both public and private goods. The government's involvement in the provision of private goods provides it with information about individuals' private good purchases that facilitates more efficient revenue extraction for the provision of public goods. We show that public provision of the private good improves economic efficiency under a condition that is always fulfilled under stochastic independence and satisfied for an open set of joint distributions.

The efficiency rationale for the public provision of private goods we advanced in this paper differs substantially from explanations emphasized in the existing literature, which typically rely on preferences for redistribution. Our result is also derived under considerably less stringent restrictions on the set of admissible policies than the existing literature. We do not impose any restrictions on the benchmark outcome that can be implemented under the optimal separate provision mechanism, which is in stark contrast to the existing literature where the benchmark outcome is often derived under various implicit restrictions on the market operations. The no-arbitrage restriction we require for the joint provision mechanism to improve upon the benchmark outcome in our model also accords with many publicly-provided private goods we see in reality because in-state college tuition, public health insurance, and public schools are all commodities that are difficult to resell. Thus our paper also provides an explanation of why some, but not all, private goods are publicly provided.

Finally, our analysis also exemplifies a more general point about the optimal taxation literature. Our design problem combines a simple commodity taxation problem with an excludable public good provision problem. We found that the marginal price for access to the public good for consumers that purchase the private good should be different from those who do not. Hence, our model is a stylized example where provision and user fees for the public good, and taxes on the private good must be jointly determined in order to achieve economic efficiency. Our paper thus illustrates that the standard practice of separating the question of how a given budget should be spent from the question of how a given tax revenue should be raised generates efficiency losses. 


\section{References}

[1] Adams, William James and Janet L. Yellen (1976). "Commodity Bundling and the Burden of Monopoly." Quarterly Journal of Economics, Vol. 90, No. 3, 475-498.

[2] Al-Najjar, Nabil (2204). "Aggregation and the Law of Large Numbers in Large Economies." Games and Economic Behavior, Vol. 47, 1-35.

[3] Bergstrom, Ted and Sören Blomquist (1996). "The Political Economy of Subsidized Day Care." European Journal of Political Economy, 12, Vol. 3, 443-458.

[4] Besley, Timothy and Stephen Coate (1991). "Public Provision of Private Goods and the Redistribution of Income." American Economic Review, Vol. 81, No. 4, 979-984.

[5] Blackorby, Charles and David Donaldson (1988). "Cash versus Kind, Self-Selection, and Efficient Transfers." American Economic Review, Vol. 78, No. 4, 691-700.

[6] Blomquist, Sören and Vidar Christiansen (1995). "Public Provision of Private Goods as a Redistributive Device in an Optimum Income Tax Model." Scandinavian Journal of Economics, Vol. 97, No. 4, 547-67.

[7] Blomquist, Sören and Vidar Christiansen (1999). "The Political Economy of Publicly Provided Private goods." Journal of Public Economics, 73(1), 31-54

[8] Blomquist, Sören and Vidar Christiansen (2007). "Public Provision of Private Goods and Non-Distortionary Marginal Tax Rates." mimeo, University of Uppsala.

[9] Boadway, Robin, Maurice Marchand, and Motohiro Sato (1998). "Subsidies versus Public Provision of Private Goods as Instruments for Redistribution." Scandinavian Journal of Economics, Vol. 100, No. 3, 545-564.

[10] Bruce, Neil and Michael Waldman (1991). "Transfers In-Kind: Why They Can Be Efficient and Nonpaternalistic." American Economic Review, Vol 81, No 5, 1345-1351.

[11] Borgers, Tilman and Peter Norman (2008). "A Note on Budget Balance under Interim Participation Constraints: The Case of Independent Types." forthcoming, Economic Theory.

[12] Coate, Stephen (1995). "Altruism, the Samaritan's Dilemma, and Government Transfer Policy." American Economic Review, Vol 85, No. 1, 46-57. 
[13] Cremer, Helmuth and Firouz Gahvari (1997). "In-Kind transfers, Self-Selection and Optimal Tax Policy." European Economic Review, Vol. 41, No. 1, 97-114.

[14] Epple, Dennis and Richard E. Romano (1996). "Public Provision of Private Goods." Journal of Political Economy, Vol. 104, No. 1, 57-84.

[15] Fang, Hanming and Peter Norman (2008). "Optimal Provision of Multiple Excludable Public Goods." mimeo, Duke University and UNC-Chapel Hill.

[16] Fang, Hanming and Peter Norman (2007). "To Bundle or Not to Bundle." Rand Journal of Economics, 2006, Vol. 37, No. 4, 946-963.

[17] Fernandez, Raquel and Richard Rogerson (1995). "On the Political Economy of Education Subsidies." Review of Economic Studies, Vol. 62, No. 2 ,249-262.

[18] Gahvari, Firouz and Enlinson Mattos (2007). "Conditional Cash Transfers, Public Provision of Private Goods, and Income Redistribution." American Economic Review, Vol. 97, No. 1, 491-502.

[19] Garratt, Rod and John M. Marshall (1994). "Public Finance of Private Goods: The Case of College Education." Journal of Political Economy, Vol. 102, No. 3, 566-582.

[20] Gouveia, Miguel (1997). "Majority Rule and the Public Provision of a Private Good." Public Choice, Vol. 93, No. 3-4, 221-44.

[21] Hellwig, Martin F. (2005). "A Utilitarian Approach to the Provision and Pricing of Excludable Public Goods." Journal of Public Economics, Vol. 89, No. 11-12, 1981-2003.

[22] Hellwig, Martin F (2003). "Public Good Provision with Many Participants." Review of Economic Studies, Vol. 70, No. 2, 589-614.

[23] Mailath, George and Andrew Postlewaite (1990). "Asymmetric Information Bargaining Problems with Many Agents." Review of Economic Studies, Vol. 57, No. 2, 351-368.

[24] Mas-Colell, Andreu, Michael Whinston and Jerry R. Green (1995). Microeconomic Theory. Oxford University Press.

[25] McAfee, R. Preston, John McMillan and Michael D. Whinston (1989). "Multiproduct Monopoly, Commodity Bundling, and Correlation of Values." Quarterly Journal of Economics, Vol. 104, No. 1, 371-84. 
[26] Myerson, Roger (1981). "Optimal Auction Design." Mathematics of Operations Research, Vol. 6, No. 1, 58-73.

[27] Myerson, Roger and Mark A. Satterthwaite (1982). "Efficient Mechanisms for Bilateral Trading." Journal of Economic Theory, Vol. 29, 265-281.

[28] Nichols, Albert L. and Richard J. Zeckhauser (1982). "Targeting Transfers Through Restrictions on Recipients." American Economic Review Papers and Proceedings, Vol. 72, 301-17.

[29] Norman, Peter (2004). "Efficient Mechanisms for Public Goods with Use Exclusion." Review of Economic Studies, Vol. 71, 1163-1188.

[30] Ramsey, Frank P. (1927). "A Contribution to the Theory of Taxation." Economic Journal, Vol. 37, No 145, 47-61.

[31] Riley, John and Richard Zeckhauser (1983). "Optimal Selling Strategies: When to Haggle, When to Hold Firm." Quarterly Journal of Economics, Vol. 98, No. 2, 267-289.

[32] Roberts, John (1976). "The Incentives for Correct Revelation of Preferences and the Number of Consumers." Journal of Public Economics, Vol. 6, 359-374.

[33] Samuelson, Paul A. (1958). "Aspects of Public Expenditure Theories." Review of Economics and Statistics, Vol. 40, 332-338.

[34] Schmitz, P. W. (1997). "Monopolistic Provision of Excludable Public Goods Under Private Information." Public Finance, Vol. 52, No. 1, 89-101.

[35] Stokey, Nancy L. (1979). "Intertemporal Price Discrimination." Quarterly Journal of Economics, Vol. 93, No. 3, 355-371. 